\title{
Propositional Identity and Logical Necessity
}

\author{
David B. Martens \\ Department of Philosophy \\ Auburn University* \\ dbm@ca.inter.net \\ Received by Greg Restall \\ Published March I2, 2004 \\ http://www.philosophy . unimelb.edu.au/ajl/2004 \\ (C) 2004 David B. Martens
}

\begin{abstract}
In two early papers, Max Cresswell constructed two formal logics of propositional identity, PCR and FCR, which he observed to be respectively deductively equivalent to modal logics $\mathrm{s}_{4}$ and $\mathrm{s}_{5}$. Cresswell argued informally that these equivalences respectively "give ... evidence" for the correctness of $\mathrm{s}_{4}$ and $\mathrm{s}_{5}$ as logics of broadly logical necessity. In this paper, I describe weaker propositional identity logics than PCR that accommodate core intuitions about identity and I argue that Cresswell's informal arguments do not firmly and without epistemic circularity justify accepting $\mathrm{s}_{4}$ or $\mathrm{s5}$. I also describe how to formulate standard modal $\operatorname{logics}(\mathrm{K}, \mathrm{s} 2$, and their extensions) with strict equivalence as the only modal primitive.
\end{abstract}

\section{TWO PROPOSITIONAL IDENTITY LOGICS}

Cresswell [2, 3] constructs two formal logics of propositional identity, PCR and FCR, and informally argues for the correctness of s4 and s 5 as logics of broadly logical necessity on the grounds of their respective deductive equivalence to PCR and FCR. I will describe weaker propositional identity logics than PCR that accommodate core intuitions about identity, and I will argue that Cresswell's informal arguments do not firmly and without epistemic circularity justify accepting s4 or s5. I myself will not argue for or against the correctness of s4 or s5.

${ }^{*}$ A version of this paper was presented at the 2003 Northwest Philosophy Conference at Reed College where I benefited from comments by Peter Hanks and further discussion with Anthony Anderson and Mark Hinchliff. I also received helpful comments on various versions and ancestors from Bernard Linsky, Peter Loptson, Ernest Sosa, Karen Wendling, and anonymous readers. 
PCR is PC $+\{$ ASI, R, DFI, DF2, DF3 $\}$. PC is the classical non-modal propositional calculus formulated with uniform substitution (Us) and modus ponens (мР) as basic inference rules. $T$ is an arbitrary PC-tautology. ' $=$ ' is a binary modal operator and is the only modal primitive in PCR. ' $\alpha=\beta$ ' is read as 'that $\alpha$ is the very same proposition as that $\beta^{\prime} \mathrm{I}^{2}$

(ASI) $\vdash(p=q) \supset(\alpha \supset \beta)$, provided that $\alpha$ differs from $\beta$ only in having $p$ in some of the places where $\beta$ has $q$.

(R) If $\vdash(\alpha \equiv \beta)$ then $\vdash(\alpha=\beta)$.

(DFI) $\square \alpha={ }_{\mathrm{df}}(\alpha=T)$

(DF2) $(\alpha \leftrightarrow \beta)={ }_{\mathrm{df}} \square(\alpha \equiv \beta)$

(DF3) $\diamond \alpha={ }_{\mathrm{df}} \sim \square \sim \alpha$

PCR has theorems (LD, L) formally expressing the contentious metaphysical view that (M) strict equivalence is propositional identity and logical necessity is identity with a tautology 3

(LD) $(p=q)=(p \leftrightarrow q)$

(L) $\square p=(p=\top)$

PCR's sole axiom, ASI, is a principle of substitutivity (or indiscernibility) of identical propositions and PCR has theorems (PII-PI3) affirming the reflexivity, symmetry, and transitivity of propositional identity 4

(PII) $p=p$

(PI2) $(p=q) \supset(q=p)$

II use "PCR" and other names of logical systems with deliberate ambiguity. In one sense, for example, "PCR" names a logic, that is, a set of theses (axioms and theorems). In another sense, "PCR" names a particular basis or axiomatization of that logic, that is, a particular set of axioms, basic inference rules, and definitions, whose closure is PCR (first sense). Context will generally resolve this ambiguity but I will sometimes specify a sense explicitly.

${ }^{2}$ For my own convenience, I have altered Cresswell's notation and some propositions' names, and added some definitions. I generally use '=' as an operator (at Prior's suggestion; see I6, p. I90], for example) rather than as a relational predicate. I do sometimes use ' $=$ ' as a relational predicate in the meta-language or in first-order object-language expressions, but such non-operator uses of ' $=$ ' are clear from context.

3'“What are the conditions for propositional identity?' ... is a language-independent metaphysical question whose answer demands an analysis ... of the notion of a proposition" $[5$, p. 45]. That strict equivalence is propositional identity and necessity is identity with a tautology is implied by the views that (a) propositions are sets of worlds while (b) necessity is truth in all possible worlds [5] pp. 24, 39].

${ }^{4}$ Proofs of formal results reported in this paper are not difficult and are left as exercises for the reader. 
(PI3) $((p=q) \wedge(q=r)) \supset(p=r)$

Cresswell proves that PCR is deductively equivalent to $\mathrm{S} 4$ when the latter is extended to formally express (M). $\mathrm{S}_{4}$ is $\mathrm{PC}+\{\mathrm{K}, \mathrm{T}, \mathbf{4}, \mathrm{N}, \mathrm{DF} 2, \mathrm{DF} 3\} .4$ is the controversial characteristic thesis of $\mathrm{s} 4$. ' $\square$ ' is the only modal primitive in this formulation of $s_{4}$.

(к) $\square(\mathrm{p} \supset \mathrm{q}) \supset(\square p \supset \square \mathrm{q})$

(T) $\square p \supset p$

(4) $\square p \supset \square \square p$

(N) If $\vdash \alpha$ then $\vdash \square \alpha$.

Cresswell proves that PCR is deductively equivalent to $\mathrm{S}_{4}+\mathrm{DF} 4$ [2, p. I92].

$\left(D_{4}\right)(p=q)=_{d f}(p \leftrightarrow q)$

$\mathrm{S}_{4}+\mathrm{DF} 4$ has as theorems LD, L, ASI, and PII-PI3. (M) is also well-expressed by another extension of $\mathrm{s}_{4}$, that is, by $\mathrm{s}_{4}+\mathrm{LD}^{\prime}$.

$\left(\mathrm{LD}^{\prime}\right)(\mathrm{p}=\mathrm{q}) \equiv(\mathrm{p} \leftrightarrow \mathrm{q})$

Since substitution of proved material equivalents ( $\mathrm{EQ}$ ) is available even in $\mathrm{K}$, where $\mathrm{K}$ is $\mathrm{S}_{4}-\{\mathbf{T}, \mathbf{4}\}$, Cresswell's proof with trivial modifications establishes that PCR is deductively equivalent to $\mathrm{S}_{4}+\mathrm{LD}^{\prime}$.

Appealing to the formal results he describes, Cresswell argues informally for $\mathrm{s} 4$ as a logic of broadly logical necessity.

The interesting point about PCR however is that ASI is simply the identity schema and $\mathrm{R}$ also seems to contain no reference to modality. The equivalence of PCR and S4 would seem to give further evidence for the view ... that where [' $\square$ '] means, 'It is informally provable that' then $\mathrm{s}_{4}$ is the system which captures its meaning [2 p. 195].

In other words, Cresswell does not rest justification for $\mathrm{s} 4$ merely on variable intuitions about controversial principles explicitly about necessity [6, p. 29]; [7. pp. 5I-52]. Instead, he adopts metaphysical view (M) and then aims to rest justification for $\mathrm{s} 4$ on what I will call core intuitions about identity, that is, on intuitions about identity that are both uniformly firmer and more widespread than intuitions explicitly about controversial elements of s4 or s5. Philosophers disagree about 4, for example, but no one doubts that identity is somehow reflexive, symmetrical, and transitive or that identicals are somehow substitutable.

Cresswell comes later to think that there is a further firm intuition about identity that PCR does not accommodate. So he extends PCR to FCR accordingly. FCR is PCR+NI. $\perp$ is an arbitrary PC-contradiction. 
(NI) $((p=q) \supset \perp) \supset((p=q)=\perp)$

Cresswell observes that FCR is deductively equivalent to $\mathrm{s} 5$ when the latter is extended to formally express (M). $\mathrm{s}_{5}$ is $\mathrm{T}+\mathrm{E}$, where $\mathrm{T}$ is $\mathrm{s}_{4}-\mathbf{4}$.

(E) $\diamond p \supset \square \diamond p$

FCR is deductively equivalent to $\mathrm{S} 5+\mathrm{DF} 4$ and to $\mathrm{S} 5+\mathrm{LD}^{\prime}$. Since $\mathrm{FCR}$ is justified as a logic of propositional identity and FCR is deductively equivalent to $\mathrm{s} 5$, Cresswell informally argues, $\mathrm{s} 5$ is justified as a logic of broadly logical necessity [3, p. 29I].

NI apparently is motivated by an intuition that all non-identities are necessary. The principle that non-identical propositions are necessarily non-identical perhaps is formalized more clearly by PI 4 than by NI.

(PI4) $\sim(p=q) \supset \square \sim(p=q)$

PI 4 is deductively equivalent to $\mathrm{E}$ and to $\mathrm{NI}$ in both $\mathrm{T}+\mathrm{DF} 4$ and $\mathrm{T}+\mathrm{LD}^{\prime}$, and so in both $\mathrm{S}_{4}+\mathrm{DF}_{4}$ and $\mathrm{s}_{4}+\mathrm{LD}^{\prime}$.5

\section{JUSTIFYING IDENTITY PRINCIPLES}

Intuitions about identity do bear on the acceptability of $\mathrm{s}_{4}$ and $\mathrm{s}_{5}$. However, some intuitions have less epistemic potency than others and some more readily than others interact epistemically with broader philosophical considerations.

For one thing, relatively controversial principles about identity are not supported by core intuitions about identity but only by what I will call peripheral intuitions about identity, that is, by intuitions that are either not uniformly firmer or not more widespread than intuitions explicitly about controversial elements of $\mathrm{s}_{4}$ or $\mathrm{s5}$. For example, the principles that all identities and nonidentities are necessary are, like 4 and E, controversial principles explicitly about necessity. So Cresswell's informal argument for 55, which rests on the intuitions behind $\mathrm{NI}$, does not firmly and non-circularly justify acceptance of s5.

The inability of peripheral intuitions about identity to firmly justify controversial principles about necessity is aggravated by the potential such intuitions have to be defeated by the epistemic force of an otherwise well-justified metaphysical view ${ }^{6}$ For example, not all identities and non-identities of in-

\footnotetext{
${ }^{5}$ By " $\alpha$ is deductively equivalent to $\beta$ in $S$ ", I mean " $S+\alpha$ is deductively equivalent to $S+\beta$ ".

${ }^{6}$ Many metaphysicians take seriously views according to which identity is or is not relative, relevant, temporary, contingent, occasional, vague, or indeterminate in ways that variously augment or undermine the justification for identity principles that otherwise are more or less plausible intuitively. For representative discussions, see the references in notes 7 and 8, below.
} 
dividuals are necessary if counterpart theory is true.7 So, if counterpart theory were otherwise well-justified, the peripheral intuitive support for the principles that identities and non-identities are necessary might be defeated. The general point applies also to propositional identity. For example, if any metaphysical view were otherwise well-justified that implies both (a) theses of contingent identity and diversity of individuals and (b) a thesis of wide content (say, counterpart theory plus Russellian propositions), then the principles of the necessity of propositional identity and diversity might be undermined. For argument's sake, represent the proposition that $p$, that you and I are identical, as $\langle y o u, m e, x$ is identical with $y$ 〉 and represent the proposition that $q$, that I am self-identical as $\langle m e, m e, x$ is identical with $y\rangle$. That $p$ is not the very same proposition as that $\mathrm{q}$, since they are wholes whose parts are not all shared. However, in a world in which you and I have the same counterpart, the propositions that $p$ and that $q$ also have the same counterpart. Say that our counterpart in one such world is Bloggs. Represent the proposition that $r$, which is the counterpart there of the propositions that $p$ and that $q$, as $\langle$ Bloggs, Bloggs, $x$ is identical with $y$. That $r$ is the very same proposition as that $r$, so it is possible that that $p$ is the very same proposition as that $q$. So, apparently, diverse propositions are not necessarily diverse if such a metaphysical view is true. Nor, by analogous reasoning, are identical propositions necessarily identical.

Like peripheral intuitions, core intuitions about identity also interact epistemically with broader philosophical considerations. Core intuitions do firmly support the uncontroversial but vague principles that identity is somehow reflexive, symmetrical, and transitive and that identicals are somehow substitutable. However, each of these vague informal principles can be given alternative but nonequivalent formal expressions, none inheriting more apparent presumptive justification than the others from the intuitions behind their vague parent. No choice of one formal expression rather than another of a vague informal principle can be justified merely by the intuitions backing the informal principle—-broader philosophical considerations must be brought to bear ${ }^{8}$

\footnotetext{
${ }^{7} x$ (this plastic) and $y$ (this dishpan) are identical, but there is a world in which the counterpart of $x$ is a wastebasket and the counterpart of $y$ is made of different plastic, so $x$ and $y$ are not necessarily identical. You and I are non-identical, but there is a world in which we have the same counterpart, so we are not necessarily non-identical. See Lewis [12, I3]. For related discussions, see Kripke [IO, II], Marcus [I4], and Noonan [I5].

${ }^{8}$ Kremer notes that "the principle of symmetry has ... non-equivalent forms", that "With transitivity, we have even more choices", and that there are "various forms of indiscernibility and substitution" [9. pp. 200, 20I]. He also usefully points out that the precise range of alternatives available for expressing a given informal identity principle within a given formal system depends on the formal resources available in the system. Perhaps it is not quite right, though, to say (where ' $=$ ' is a relational predicate, not a modal operator) that "The only version of reflexivity is (REFL) $x=x "$ [9 p. 200]. Perhaps such formal principles as the necessity of self-identity of individuals ([REFL'] $\square x=x$ ) and the essentiality of self-identity of individuals ( $\left[\mathrm{REFL}^{\prime \prime}\right] x$ exists $\supset \square x=x$, or [REFL $\left.{ }^{\prime \prime \prime}\right] \square(x$ exists $\supset x=x)$ ) may also be regarded as alternative formal expressions of the principle that identity is reflexive.
} 
For example, in the formal vocabulary of standard modal predicate logic (where ' $=$ ' is a relational predicate, not a modal operator), I2 and I 2 ' each express the vague principle that identicals are somehow substitutable.

(I2) $x=y \supset(\alpha \supset \beta)$, provided that $\alpha$ differs from $\beta$ only in having free $x$ in some of the places where $\beta$ has free $y$.

(I2 $\left.{ }^{\prime}\right) x=y \supset(\alpha \supset \beta)$, provided that $\alpha$ differs from $\beta$ only in having free $x$ in some of the places, not in the scope of any modal operator, where $\beta$ has free $y$.

However, I2 does while I $2^{\prime}$ does not imply that all identities are necessary [6, p. I95]; [7, p. 334]. So acceptance of I $2^{\prime}$ rather than I 2 may accommodate both the intuitive precariousness of the principle that all identities are necessary and the intuitive fundamentality of the principle that identicals are somehow substitutable. Again, the general point applies also to propositional identity. For example, ASI, PI5, and PI 6 each express the vague principle that identical propositions are somehow substitutable.

(PI5) If $\vdash(\gamma=\delta)$ then $\vdash(\alpha \supset \beta)$, provided that $\alpha$ differs from $\beta$ only in having $\gamma$ in some of the places where $\beta$ has $\delta$.

(PI6) $\vdash(p=q) \supset(\alpha \supset \beta)$, provided that $\alpha$ differs from $\beta$ only in having $p$ in some of the places, not in the scope of any modal operator, where $\beta$ has q.

However, while AS I implies that (P I 7 ) all propositional identities are necessary, neither PI5 nor PI6 implies PI7.

$(\operatorname{PI})(p=q) \supset \square(p=q)$

So acceptance of either PI5 or PI6 rather than ASI may accommodate both the intuitive precariousness of the principle that all propositional identities are necessary and the intuitive fundamentality of the principle that identical propositions are somehow substitutable.

So, if there are weaker propositional identity logics than PCR that accommodate core intuitions about identity, then a choice of one of those logics over the others cannot be justified merely by those core intuitions and Cresswell's informal argument for $\mathrm{s} 4$ does not firmly and non-circularly justify acceptance of $\mathrm{s}_{4}$.

\section{Normal propositional identity LOGiCS}

There are propositional identity logics that are weaker than PCR and in which propositional identity is somehow reflexive, symmetrical, and transitive, and identical propositions are somehow substitutable. 
PCR is such a spartan basis for $\mathrm{s}_{4}$ that it is hard to see how to weaken necessity in the system. Things are easier if one begins with a more usual basis for $\mathrm{s}_{4}$, extended to express (M). I will begin with $\mathrm{s}_{4}+\mathrm{LD}^{\prime}$, which has as theorems that necessity is both identical with and materially equivalent to identity with a tautology $\left(\mathrm{L}, \mathrm{L}^{\prime}\right)$.

$\left(\mathrm{L}^{\prime}\right) \square p \equiv(\mathrm{p}=\top)$

The basis of $\mathrm{s}_{4}+\mathrm{LD}^{\prime}$ is easily modified so propositional identity is the only modal primitive. Let $\mathrm{s}_{4}=$ be $\mathrm{PC}+\left\{\mathbf{K}^{=}, \mathbf{T}^{=}, \mathbf{4}^{=}, \mathrm{N}^{=}, \mathrm{LD}^{\prime \prime}, \mathrm{DFI}, \mathrm{DF} 2, \mathrm{DF} 3\right\}$.

$\left(\mathbf{k}^{=}\right)((p \supset q)=\top) \supset((p=T) \supset(q=T))$

$\left(\mathrm{r}^{=}\right)(\mathrm{p}=\top) \supset \mathrm{p}$

$\left(\mathbf{4}^{=}\right)(p=\top) \supset((p=\top)=\top)$

$(\mathrm{N}=)$ If $\vdash \alpha$ then $\vdash(\alpha=\top)$.

$\left(\mathrm{LD}^{\prime \prime}\right)(\mathrm{p}=\mathrm{q}) \equiv((\mathrm{p} \equiv \mathrm{q})=\top)$

The bases of $\mathrm{s}_{4}+\mathrm{LD}^{\prime}$ and $\mathrm{s}_{4}=$ share $\mathrm{PC}, \mathrm{DF} 2$, and $\mathrm{DF} 3 . \mathrm{S}_{4}+\mathrm{LD}^{\prime}$ also has the other elements of the basis of $\mathrm{s}_{4}{ }^{=}$, since $\mathrm{K}^{=}, \mathrm{T}^{=}, 4^{=}, \mathrm{N}^{=}$, and $\mathrm{LD}^{\prime \prime}$ are all provable by $\mathrm{L}^{\prime}$. And $\mathrm{s}_{4}=$ has the other elements of the basis of $\mathrm{s}_{4}+\mathrm{LD}^{\prime}$, since $\mathbf{K}, \mathbf{T}, \mathbf{4}, \mathrm{N}$, and $\mathrm{LD}^{\prime}$ are all provable by DFI. So $\mathrm{S}_{4}{ }^{=}$is deductively equivalent to $\mathrm{S}_{4}+\mathrm{LD}^{\prime}$ and so to PCR.

$\mathrm{S}_{4}=$ is a less spartan basis than PCR for $\mathrm{S}_{4}$ and necessity in that less spartan basis is easily weakened. Let $\mathrm{T}^{=}$be $\mathrm{s}_{4}=-4 . \mathrm{T}^{=}$is deductively equivalent to $\mathrm{T}+\mathrm{LD}^{\prime}$ and to $\mathrm{T}+\mathrm{DF} 4$. Both $\mathrm{T}^{=}$and $\mathrm{s}_{4}=$ are logics in which propositional identity is the only modal primitive. But necessity in $\mathrm{T}^{=}$( $\mathrm{T}$-necessity) is weaker than necessity in $\mathrm{s}_{4}=$ ( $\mathrm{s} 4$-necessity). A spectrum of propositional identity logics is constructible in an obvious way. Each system in the spectrum has propositional identity as its only modal primitive, but necessity varies in strength from system to system, from K-necessity to s5-necessity. So, for example, let $\mathbf{K}^{=}$be $\mathbf{T}^{=}-\mathbf{T}^{=}, \mathbf{B}^{=}$be $\mathbf{T}^{=}+\mathbf{B}^{=}$, and $\mathrm{s}_{5}=$ be $\mathbf{T}^{=}+\mathbf{E}^{=} 9$

${ }^{9}$ Each of $\mathrm{K}, \mathrm{T}, \mathrm{B}, \mathrm{s}_{4}$, and $\mathrm{s}_{5}$ (and their extensions) can be given bases with strict equivalence as the only modal primitive (sep-bases), as follows. From the basis for the corresponding propositional identity logic, delete both $\mathrm{LD}^{\prime \prime}$ and DF2, replace DFI with DF5, and uniformly replace '=' throughout the basis with ' $\leftrightarrow$ '.

(DF5) $\square \alpha==_{\mathrm{df}}(\alpha \leftrightarrow \top)$

Any modal logic that has both EQ and $\mathrm{L}^{\prime \prime}$ and that has a basis with necessity as the only modal primitive (an $n p$-basis) can also be given an sep-basis by deleting any pre-existing definition of ' $\leftrightarrow$ ' from the np-basis; then, for all $\alpha$, uniformly replacing $\ulcorner\square \alpha\urcorner$ throughout the basis with $\ulcorner\alpha \leftrightarrow \top\urcorner$; then adding DF5.

$\left(\mathrm{L}^{\prime \prime}\right) \square \mathrm{p} \equiv(\mathrm{p} \leftrightarrow \top)$ 
$\left(\mathbf{B}^{=}\right) p \supset(\sim(\sim p=\top)=\top)$

$\left(\mathbf{E}^{=}\right) \sim(\sim p=\top) \supset(\sim(\sim p=\top)=\top)$

Call a propositional identity logic normal if it can be formulated on a basis that contains the basis just given for $\mathrm{K}={ }^{10}$ Every normal propositional identity logic has $\mathbf{K}^{=}$, is closed under Us, MP, and $\mathrm{N}^{=}$, and has all the following: $\mathrm{L}, \mathrm{L}^{\prime}$, LD, LD', LD', PII, PI2, PI3, PI5, R. In other words, every normal propositional identity logic formally expresses metaphysical view (M): strict equivalence is propositional identity $\left(\mathrm{LD}, \mathrm{LD}^{\prime}, \mathrm{LD}^{\prime \prime}, \mathrm{R}\right)$, and necessity is identity with a tautology $\left(\mathrm{L}, \mathrm{L}^{\prime}\right)$. And every normal modal propositional identity logic accommodates core intuitions about identity: propositional identity is somehow reflexive (PII), symmetric (P I2), and transitive (PI3), and identical propositions are somehow substitutable (PI5).

Differences between stronger and weaker normal propositional identity logics correspond to the presence or absence of specific theorems or rules about propositional identity that are not imposed by core intuitions about identity.

$\mathrm{T}^{=}$and $\mathrm{K}=$ differ in having or lacking PI6, which is deductively equivalent to $\mathrm{T}$ in $\mathrm{K}=$. PI 6 says that identical propositions, whether or not their identity is provable, are substitutable indiscernibly in non-modal (extensional) contexts. This contrasts with PI5, according to which provably identical propositions are substitutable indiscernibly in all contexts in theorems. That is, PI5 and PI6 do not permit all the same substitutions. $\mathrm{T}^{=}$has both PI5 and PI6, while $\mathrm{K}=$

The preceding method can be used to give sep-bases for $\mathrm{K}$ and its extensions. Huntington $[8$ provides an sep-basis for s2. Though Hughes and Cresswell [6, p. 296] attribute DF5 to him, Huntington [8, p. 5] actually uses DF 6 , where '*' is a unary modal operator and '* $\alpha$ ' is read 'it is impossible that $\alpha$.

$(\mathrm{DF} 6) * \alpha={ }_{\mathrm{df}}(\alpha \leftrightarrow \perp)$

DFI is given by Bronstein and Tarter [I, p. 307], who are mentioned by Hughes and Cresswell [6, p. 297, note 334]. DF I easily suggests DF5. In his formulation of s2, Huntington also uses DF7.

$(\mathrm{DF} 7)(\alpha \rightarrow \beta)=_{\mathrm{df}} *(\alpha \wedge \sim \beta)$

Huntington's sep-basis for s2 can be modified in obvious ways to provide sep-bases for each of s2's extensions ( $\mathrm{s}_{3}, \mathrm{~T}$, etc.). For example, adding 3 as an axiom yields $\mathrm{s}_{3}$.

(3) $(p \rightarrow q) \rightarrow(* q \rightarrow * p)$

${ }^{10}$ Call a propositional identity logic intensional if it has $\mathrm{LD}^{\prime}$. All normal propositional identity logics are intensional. If metaphysical view (M) is doubted, then-since $\mathrm{LD}^{\prime}$ is motivated by (M) - non-intensional propositional identity logics likely will be of interest and the formal distinction between ' $=$ ' and ' $\leftrightarrow$ ' will be significant. If (M) is not doubted, then $\mathrm{LD}^{\prime}$ may seem trivial, the formal distinction between ' $=$ ' and ' $\leftrightarrow$ ' may seem otiose, and any standard modal logic, normal or not, can be made to serve as a propositional identity logic simply be reading ' $\alpha \leftrightarrow \beta$ ' in that logic as 'that $\alpha$ is the very same proposition as that $\beta$ ', i.e., by adding DF 4 . If a non-normal logic so serves, it is as a non-normal propositional identity logic. 
has only PI5. So substitutivity (or indiscernibility) of identical propositions is stronger in $\mathrm{T}^{=}$than in $\mathrm{K}^{=}$.

The difference between $\mathrm{s}_{4}=$ and $\mathrm{T}^{=}$has two aspects. On one hand, it is the difference between the presence or absence of ASI, which is deductively equivalent to 4 in $\mathrm{T}^{=}$. AS I says that identical propositions, whether or not their identity is provable, are substitutable in all contexts. That is, As I permits substitutions that neither PI5 nor PI 6 permits. $\mathrm{S}_{4}=$ has ASI, PI5, and PI6, so substitutivity (or indiscernibility) of identical propositions is stronger in $\mathrm{s}_{4}=$ than in $\mathrm{T}^{=}$. But the difference between $\mathrm{T}^{=}$and $\mathrm{s}_{4}=$ is also the difference between the presence or absence of PI7, a strong principle of the necessity of propositional identity that is deductively equivalent to 4 and so to ASI in $T^{=}$.

$\mathbf{B}^{=}$and $\mathrm{T}^{=}$differ in having or lacking PI 8 , which is deductively equivalent to $\mathrm{B}$ in $\mathrm{T}^{=}$.

$($ I I 8$) \sim(p \equiv q) \supset \square \sim(p=q)$

(в) $p \supset \square \diamond p$

PI 8 is a weak principle of the necessity of propositional diversity, according to which propositions differing in truth-value are necessarily diverse.

A stronger principle of the necessity of propositional diversity is P I 4, which is deductively equivalent to $\mathrm{E}$ in $\mathrm{T}^{=}$. So $\mathrm{S}_{5}{ }^{=}$and $\mathrm{T}^{=}$differ in having or lacking PI 4 .

\section{Conclusion}

Cresswell's informal arguments show how modal logics s4 and s5 cohere with metaphysical view (M). However, those arguments do not firmly and without epistemic circularity justify accepting $\mathrm{s}_{4}$ or $\mathrm{s}_{5}$, even conditional on the acceptability of (м). Since a number of principles of propositional identity that are crucial for the justification by Cresswell's strategy even of the weaker s 4 are also principles explicitly about necessity, it is difficult to see how a justifying argument for $\mathrm{s}_{4}$ or $\mathrm{s}_{5}$ might be constructed in which considerations about propositional identity have clear epistemic priority over considerations about broadly logical necessity. Since every normal propositional identity logic both formally expresses (M) and accommodates core intuitions about identity, it is difficult to see how intuitions about propositional identity alone, unaided by broader philosophical considerations, might justify acceptance of a propositional identity logic stronger than $\mathrm{K}^{=}$; but there is anyway relatively little doubt that strong and widespread intuitions about broadly logical necessity alone tend to justify acceptance of a modal logic at least as strong as $\mathrm{T}$

\footnotetext{
${ }^{11}$ Hughes and Cresswell [6, pp. 25-30]; [7. pp. 51-52]. Even the intuitions of C. I. Lewis, whose official system was the weaker s2, are said to point rather in the direction of $\mathrm{T}$ [4, p. 204].
} 


\section{REFERENCES}

[I] Daniel J. Bronstein and Harry Tarter, Review of C. I. Lewis and C. H. Langford, Symbolic Logic, in The Philosophical Reviere, vol. 43 (1934), pp. 305-309.

[2] M. J. Cresswell, 'Another Basis for s4', Logique et Analyse, vol. 8 (1965), pp. I9I-195.

[3] M. J. Cresswell, 'Propositional Identity', Logique et Analyse, vol. Io (1967), pp. $283-292$.

[4] M. J. Cresswell, 'The Interpretation of Some Lewis Systems of Modal Logic', Australasian fournal of Philosophy, vol. 45 (1967), pp. 198-206.

[5] M. J. Cresswell, Logics and Languages, Methuen, London, 1973.

[6] G. E. Hughes and M. J. Cresswell, An Introduction to Modal Logic, Methuen, London, 1968.

[7] G. E. Hughes and M. J. Cresswell, A New Introduction to Modal Logic, Routledge, London, 1996.

[8] Edward V. Huntington, 'Postulates for Assertion, Conjunction, Negation, and Equality', Proceedings of the American Academy of Arts and Sciences, vol. 72 (1937), pp. I-44.

[9] Philip Kremer, 'Relevant Identity', fournal of Philosophical Logic, vol. 28 (1999), pp. 199-222.

[ıo] Saul Kripke, 'Identity and Necessity', in Milton K. Munitz, ed., Identity and Individuation, New York University Press (1971), pp. 135-164.

[II] Saul Kripke, Naming and Necessity, Harvard University Press, I980.

[I2] David Lewis, 'Counterpart Theory and Quantified Modal Logic', in Michael J. Loux, ed., The Possible and the Actual, Cornell University Press (1979), pp. IIO-I28.

[13] David Lewis, On the Plurality of Worlds, Blackwell, Oxford, 1986.

[14] Ruth Barcan Marcus, Modalities, Oxford University Press, 1993.

[15] Harold Noonan, ed., Identity, Dartmouth Publishing, Aldershot, 1993.

[16] A. N. Prior, 'Is the Concept of Referential Opacity Really Necessary?', Acta Philosophica Fennica, vol. I6 (1963), pp. I89-199. 
The Australasian fournal of Logic (ISSN 1448-5052) disseminates articles that significantly advance the study of logic, in its mathematical, philosophical or computational guises. The scope of the journal includes all areas of logic, both pure and applied to topics in philosophy, mathematics, computation, linguistics and the other sciences.

Articles appearing in the journal have been carefully and critically refereed under the responsibility of members of the Editorial Board. Only papers judged to be both significant and excellent are accepted for publication.

The journal is freely available at the journal website at

$$
\text { http://www.philosophy.unimelb.edu.au/ajl/ }
$$

All issues of the journal are archived electronically at the journal website.

SUBSCRIPTIONS Individuals may subscribe to the journal by sending an email, including a full name, and email address and a postal address to the managing editor at ajl-editors@unimelb.edu.au Subscribers will receive email abstracts of accepted papers to an address of their choice. For institutional subscription, please email the managing editor at ajl-editors@unimelb.edu.au

Complete published papers may be downloaded at the journal's website at http: //www.philosophy.unimelb.edu.au/ajl/ The journal currently publishes in pdf format.

Submission The journal accepts submissions of papers electronically. To submit an article for publication, send the $\mathrm{LT}_{\mathrm{E}} \mathrm{X}$ source of a submission to a member of the editorial board. For a current list of the editorial board, consult the website.

The copyright of each article remains with the author or authors of that article. 